\title{
Collaborative teacher training through telematics
}

\author{
Giampaolo Chiappini, Augusto Chioccariello and Camillo Gibelli \\ Istituto per la Matematica Applicata, Consiglio Nazionale delle Ricerche, Via de Marini 6, \\ 16149 Genova, Italy. chiappini@ima.ge.cnr.it \\ Istituto per le Tecnologie Didattiche, Italy. \\ Don Milani Lower Secondary School, Italy.
}

Keywords: distance teacher training, computer supported co-operative learning, experience-based learning, collaborative training

\begin{abstract}
The traditional approach to in-service teacher training is based on a transmissive model and on a hierarchical relationship between experts and trainees. By contrast, our work centres on collaborative training in workgroups and experience-based learning. In our model, knowledge building is the result of trainees interacting with an environment; the creation of interactive trainee communities dedicated to projects conducted in schools stimulates the emergence of shared knowledge. In this framework, we have developed a computer network infrastructure that integrates communication and collaboration, providing an efficient solution to distance workgroup activities in teacher training. Both the methodology and the tools developed are currently being tested in a national project funded by the Italian Ministry of Education.
\end{abstract}

\section{INTRODUCTION}

Reforms currently being introduced in the Italian education system (OECD 98) are aimed at fundamentally restructuring the school cycle and developing school autonomy, innovations that are significant from both the technological and curricular viewpoints. In order for these reforms to take effect, the Italian education system must be ready not so much to modernise but rather to rebuild itself. This means being able to redefine the cultural and epistemological framework, trying out new models for the organisation of teaching, and updating contents and teaching methods. 
In order to be effective, any change made within individual schools must build upon the educational developments already actuated within the school itself. In addition, prior consideration must be made about the nature of problems arising in educational management and about the nature of educational and organisational variables involved in each problem. This process cannot be imposed bureaucratically from the outside, entailing as it does the re-elaboration of previous experience and, at the same time, planning and decision-making regarding changes in the system of constraints surrounding the teaching/learning process. Reform requires all the actors within the education system to become involved and take responsibility. The underlying objective of the project described in this paper is to foster schools' educational and organisational autonomy. This is to be done using network technology to improve communication and collaboration between the various actors in the education system, helping schools to re-elaborate previous projects and develop collaborative planning skills.

\section{ASSUMPTIONS AND CONTEXT OF THE RESEARCH}

Copernicus is a Ministry of Education training project designed to foster the autonomy of schools. It is based around six centres located throughout Italy and involves collaboration with universities and research organisations. Each centre gathers together twelve primary, lower- and upper-secondary schools, and within each school there are five representatives (the principal, three teachers and head of administration).

Each centre has its own specific research and training theme regarding school autonomy. The theme in Genoa is 'distance co-operation and collaboration to support school autonomy'. The pedagogical philosophy guiding the development of our segment of the Copernicus network is to promote an active, participatory and collaborative learning process, as defined by socio-cultural philosophy (Vygotsky 1962; Cole 1985; Engeström 1987). Copernicus is a collaborative training project designed to transfer knowledge and develop skills through group interaction. This approach to training is in stark contrast with the transmissive model that still dominates present-day schooling in Italy. The training model adopted in the Genoa section of the Copernicus network is based on four assumptions.

- Recent pilot projects have led to the development of professional skills within schools and these can and must be mobilised and used for training within those same schools.

- Actors in the education system (directors, teachers and administrative staff) must be considered active players in their own training process, 
and training itself should be seen as a social process of construction and appropriation of new ideas and methods. Training ought to be based on the description, comparison and re-elaboration of the experiences conducted in schools, and on assisted testing of the new educational and organisational approaches shared by all.

- The research contribution must no longer be limited to the transmission of academic knowledge or the dissemination of research results. A new kind of relationship between research, training and educational/administrative innovation must begin to take shape. The first step is the realisation that there are no pre-set roles, authorities or responsibilities in the relationship between the research world and schools involved in training; these must be subject to ongoing negotiation (Bottino and Chiappini 1998).

- Technology in general and computers and computer networks in particular can be used to actuate the training processes underlying the above assumptions. Nowadays, collaborative training in workgroups and experience-based learning are pedagogical training methods that can be supported and integrated using ICT. The former promotes learning through active communication among members in a workgroup, while the latter consolidates training concepts through the description, comparison and re-elaboration of experiences developed in schools.

\section{THEORETICAL FRAMEWORK OF THE RESEARCH}

Underlying our experience is the conviction that in order to foster collaborative training in workgroups and experience-based learning, the computer/network infrastructure adopted for the training course must provide for the construction of a social space that satisfies the professional and training needs of those involved.

The notion of social space is taken from anthropology and has been elaborated by Fortes: "An individual's social space is a product of that segment of the social structure and that segment of the habitat with which he or she is in effective contact. To put it in another way, the social space is the society in its ecological setting seen from the individual's point of view. The individual creates his social space and is in turn formed by it. On the one hand, his range of experiences and behaviour are controlled by his social space, and on the other, everything he learns causes it to expand and become more differentiated. In the lifetime of the individual it changes pari passu with his psycho-physical and social development... In the evolution of an individual's social space we have a measure of his educational development." (Fortes 1973). 
For our purposes the social space can be defined as the area where trainees interact with groups of colleagues in a learning-by-doing process. Engaging in activities, the trainees encounter and help elaborate meanings that characterise the social culture of the group. In this framework, an information and communication structure (ICS) can help to shape the trainees' social space if they recognise it as a useful tool for their production and communication activities. In other words, an ICS is useful to the trainees if it can support the motivational, social and interpersonal process of the workgroup and create three things: a concrete interdependence among the members of a group performing a task; a commitment to mutual assistance; and responsibility for the group and its objectives. Only under these conditions can an ICS provide "the crucial match between a support system in the social environment and an acquisition process in the learner" (Bruner 1985), i.e. contribute to expand and differentiate the individual's social space.

\section{COLLABORATIVE LEARNING IN WORKGROUPS}

Our experience underlines the strict correlation between evolution in the ICS made available to the trainees from one phase to the next, and the characteristics of the social space that develops in the training context with the mediation of the technological tools on offer. Three phases can be identified.

\subsection{Phase 1: Contact}

This can be defined as the contact phase, that of exploring the potential of network-based social interaction for setting up discussion groups.

At the outset of our training programme, the actors' competence in the use of computer and network technology differed enormously, as did the computer equipment available in each school. The upper-secondary schools had computers linked up in networks, whereas in the primary schools computer labs had only been up and running for a few months, and Internet connections were still being installed. None of the schools had previous experience with ICT-based training courses.

The technology employed in this early phase was email and mailing lists. At this stage the groups were merely virtual, having no actual identity. The communication structure was not arranged according to the groups' specific communication requirements but was general in nature, designed to foster interpersonal contact and exploration of the potential that multipoint network communication offers. The shift towards increasingly 'creative' 
output reveals that individuals are beginning to see themselves as group members. From the very outset, participants are called upon not just to share their experience with others, but to re-evaluate that experience and work in collaboration to formulate new responses and new solutions to the problems faced. While there is growing discussion at this stage on various issues of interest to the participants, it gradually becomes clear that the communication structure on offer is inadequate. It is too general and thus unable to meet the communication demands made of it.

At the same time, the effort of researchers focuses increasingly on the design of new environments, tailoring them to the particular needs of each group as it emerges from its anonymity and seeks out new technological solutions. In this loose beginning, hints emerge of new communication possibilities. In some cases, network interaction is uneven, suffering from structural and organisational difficulties.

As things progress, the majority of participants begin to demonstrate an effective need for an improved communication environment, and some even play a part in its design. At the same time, participant identity begins to take shape through the setting of objectives and issues for collective online discussion. However, we should note that even at this stage some of the participants are incapable of joining in productively.

\subsection{Fhase 2: Growth of group identity}

In this phase, growth in group identity is reflected in the multiplicity of common objectives and more advanced exchanges between participants. Group development reaches completion in all its facets, and the need arises for a more organised space to manage communication within the group, to control group dynamics and to trace the thread of discussion. In addition, group members need to have some way of retracing the group's communication history in order to reflect on the group's activity and at the same time discuss and compare viewpoints and solutions deriving from other experiences. For this purpose, a specially designed environment for communication and network discussion is provided. This new communication tool was created using Lotus Notes, and entails the construction of eight mailing lists that can be accessed via a simple interface. All participants are free to browse any of the lists on a read-only basis, while only those who have chosen to subscribe to a given list can exercise writing privileges. The various list functions are described below.

Together with standard computer conferencing functions, the environment features the possibility to manage the breakdown of roles among group members and non-members. In addition, participants can store messages using either a commonly agreed classification scheme or freely 
chosen Keywords. This message classification is personal and therefore cannot be seen by other participants, not even by fellow group members. The introduction of the new communication structure calls for changes in groupware management. Two new roles are created: the group co-ordinator, chosen by their fellow group members, and a facilitator appointed from among the project staff.

Table 1. Copernicus conferencing system functions

\begin{tabular}{|c|c|c|c|}
\hline $\begin{array}{l}\text { Contribute } \\
\text { (group members) }\end{array}$ & $\begin{array}{l}\text { Contribute } \\
\text { (members of other } \\
\text { groups) }\end{array}$ & Read & Classify \\
\hline $\begin{array}{l}\text { Send a message } \\
\text { Reply to a message }\end{array}$ & Send a message & $\begin{array}{l}\text { Sort messages by: } \\
\text { - date } \\
\text { - author } \\
\text { - thread }\end{array}$ & $\begin{array}{l}\text { According to a } \\
\text { shared } \\
\text { taxonomy }\end{array}$ \\
\hline $\begin{array}{l}\text { Attach files to a } \\
\text { message }\end{array}$ & & $\begin{array}{l}\text { List: } \\
\text { - unread messages } \\
\text { - non-members' } \\
\text { messages } \\
\text { - message readers }\end{array}$ & Free text \\
\hline
\end{tabular}

The new opportunities offered by the communication structure together with the new groupware management permitted the various groups in Copernicus to bring their objectives into sharper focus. A balance was struck between requirements, viewpoints, different relationship forms and communication modes, all directed towards the common goal of bringing innovation and enhancement to the experiences undertaken in schools. In this respect, we note that while there was overall improvement in the quantity and quality of message exchange, reference to previous experiences remained somewhat in the background, even though documents were at hand to be sent as attachments. Nevertheless, at this stage there was no common model for documenting the experiences. This made it difficult for the participants to get a feel for the various experiences, as they did not have the means to take them apart and understand fully the reasons, methods and conditions behind their creation, not to mention the problems in their management. Consequently, group members discussed and compared their knowledge, values and personal judgement on an individual basis, rather than setting the debate within the described experiences in order to reelaborate them. 


\subsection{Phase 3: Re-elaboration of the experiences}

This can be described as the phase of full maturity in group identity, where the experiences conducted in schools were re-elaborated and improved using the new information and communication tool, which offers the possibility of integrated communication and production.

The intense social interaction that developed in the mailing lists during Phase 2 has led to a new embodiment of common needs, ones that cannot find an effective response through use of the communication tools in play. This new embodiment has opened the way to a structured environment for the free formation of working groups so that the experiences carried out in schools can be discussed. This should lead to testing in a social context and consequent re-elaboration of the experiences themselves, hopefully bringing them more closely into line with actual training needs in education. It should be pointed out here that this new embodiment is consistent with the assumptions made by the project staff and, more importantly, that it is the result of expansion and development of the participants' social space. The latter is an outcome of the vigorous online exchange of opinion and debate that took place in earlier phases. Hence, they were not needs imposed from outside, but rather those arising from the social structure within the training process in Phases 1 and 2.

All this led to the remodelling of the information and communication structure to be used by participants. This task was performed by the project staff, who drew on the latest groupware management systems used in the business environment, tailoring their functions in response to specific project requirements. The actual systems adopted were Lotus Notes and Domino, which are used throughout the business world to allow people to work together in a faster, more efficient and productive way. We used the functionalities of these packages to develop tools we consider innovative with respect to those generally adopted in distance training for teachers. These tools broaden and further diversify the social space in response to the common needs that emerge.

Using a standard network browser, the new information structure adopted in Phase 3 allows the participants to do the following.

- Freely and autonomously create and manage the formation of workgroups that will describe experiences conducted in schools and store these in a special database. The system will automatically assign these groups reading and writing privileges for the experiences that they want to describe and share online; other Copernicus participants will only have reading rights. As they work, group members will always have the possibility to modify group composition; the responsibility for this task lies with the members themselves. 
- Fill in three specially designed forms describing an experience and create a database of HTML documents that can be seamlessly and automatically linked to those forms. Re-editing functions allow group members to update experience description in an on-going manner. The forms are designed to collect information about the background of the experience, its main characteristics and the issues that it raised. In addition, the system allows the group to link up its own experience with another in the database it deems similar.

- Consult thie database of experiences using a variety of access modes. The experience document contains links to similar experiences, together with related attachments and a list of contributors who have discussed that experience (see next point).

- Discuss the experiences in the database via mailing list, with the possibility of establishing a seamless link with the one or more experiences under discussion. These links will automatically appear in the related experience documents. The result is close integration of the experience database and the mailing list, as it is possible to call up an experience and then access any comments that have been made about it and vice versa.

- Consult the various contributions in the mailing list using a variety of access modes.

- Use functions that show who has read a message sent to a mailing list or accessed an experience in the database.

At the time of writing, Phase 3 is in full progress, so it is still too early to give a complete report on changes in the participants' social space arising from use of the information and communication structure described above. We shall thus concentrate on the qualitative changes that have occurred so far.

In this phase of Copernicus, the participants are developing the ability to construct and manage workgroups autonomously, completing the definition of their objectives and acquiring full awareness of the potential and limits of their roles. Against this background, the new environment helps to complete the gelling process between participants and the individual schools. It also fosters construction of a basic common language that clarifies ubiquitous Keywords like project and experience, words that are frequently attributed different meanings. This development is essential if the group identity is to reach full maturity.

At this stage the group is transforming itself into a production unit, i.e. an entity that can delve beyond the surface of the experiences, dismantle them and shape their future. This important result is based on the fact that the social conditions have been created whereby the input of experiences for workgroup discussion no longer reflects an individual outlook; the forms for 
analysing and describing those projects held to be important can at this stage be negotiated beforehand in relation to common goals. Finally, exchange of information about the products has given way to debate about the processes that have generated them. The greater rigidity and complexity of the discussion and storage environment (something which would have been totally out of the question at the outset of the project) is an achievement that is firmly rooted in developments in earlier phases. The tool's complexity and non-neutrality are proportional to the maturity that the subjects acquire during training, both as individuals and as groups.

In this phase, over half the participants have so far achieved these positive results: even though the others followed the group activities, their role was marginal. During this year of work, a number of obstacles were encountered along the path toward a common language and shared group objectives: the involvement of different school levels, which in Italy traditionally suffer from a lack of interaction; and the differences in professional expertise and the variety of cultural backgrounds. Our goal for the second year is to increase the percentage of people achieving the positive results described above.

\section{CONCLUSIONS}

The experience conducted thus far underlines the close interaction and reciprocal conditioning that occurs between communication technology and the professional and relational contexts of the groups engaged in training. The architectures for information gathering and communication on the one hand and the groups on the other progressively modelled themselves on each other. The information and communication tools, the contents and the forms of co-operation all developed in an ongoing manner, pooling their resources. There was no fixed hierarchy among these elements, but rather a sort of role-play in which turns were taken to put forward work plans for negotiation and testing: at one moment the information and communication structure would take the lead, at others the groups and individual trainees. The whole training path can be seen as a process whereby the training groups and collaboration environment gradually shift out of the blur into ever-sharper focus, the tools used and the social space mediated by those instruments evolving from simplicity towards complexity. 


\section{REFERENCES}

Bottino, R. M. and Chiappini, G. (1998) Teacher training: New models and tools in the era of communication technologies. In G. Davies (ed.) Proceedings of the XV IFIP Word Computer Congress, Teleteaching 98, 31st August-4th September, Vienna-Budapest.

Bruner, J. (1985) Child's talk: Learning to use language. New York:W.W. Norton.

Cole, M. (1985) The zone of proximal development: Where culture and cognition create each other. In J. V. Wertsch (ed.) Culture, communication, and cognition: Vygotskian perspectives. Cambridge, UK: Cambridge University Press.

Campo-Moltavo, E., Martinez-Orga, V., Medinilla-Martinez, N., and Meziat-Luna, D. (1998) Internet support for collaborative learning in workgroups. In G. Davies (ed.) Proceedings of the XV IFIP Word Computer Congress, Teleteaching 98, 31st August - 4th September, Vienna-Budapest.

Fortes, M. (1973) Social and psychological aspects of education in Taleleand. In J. Middeleton (ed) From child to adult: Studies in the anthropology of education. New York, Natural History Press.

Engeström Y. (1987) Learning by expanding. An activity theoretical approach to developmental research. Helsinki: Oventa Konsulktit.

OECD 98 (1998) Reviews of National Policies for Education: Italy. Paris: OECD Publication, [OECD Code 911998061P1].

Vygotsky L.S. (1962) Thought and language. Boston, MA: MIT Press.

\section{BIOGRAPHIES}

Giampaolo Chiappini is a researcher at the Institute for Applied Mathematics of the Italian National Research Council. His research interests are in educational computing, teacher training and the integration of students with disabilities. He has developed ICT-based systems for mathematics education and experimented with them in school classrooms. He has written many professional papers and participated in national and international projects on teacher education and the didactions of mathematics.

Augusto Chioccariello is a researcher at the Institute for Applied Mathematics of the Italian National Research Council. His main research interests concern both the theory and applications of ICT in education, with particular reference to constructivism in the education of pre-primary school children. He has been involved in many international projects and is currently involved in ESPIRIT CAB Project, on the development of intelligent toys for children.

Camillo Gibelli is a middle school language teacher and long-term collaborator at the Institute for Educational Technology. His main research interests concern the teaching of history and humanities with ICT. 